\title{
RODOS meteorological pre-processor and atmospheric dispersion model DIPCOT: a model suite for radionuclides dispersion in complex terrain
}

\author{
S. ANDRONOPOULOS ${ }^{1}$, E. DAVAKIS ${ }^{1}$, J.G. BARTZIS ${ }^{2}$, I. KOVALETS ${ }^{3}$
}

\begin{abstract}
The Meteorological Pre-Processor (MPP) of the Decision Support System RODOS acts as interface between the incoming meteorological data from stations and/or prognostic models and the Atmospheric Dispersion Models (ADMs) used for predicting the spread of the accidentally emitted radionuclides. The MPP includes a diagnostic Wind Field Model (WFM) to ensure mass conservation of the calculated wind field. Its output is usable by simple and complex ADMs and it is applicable for highly complex topography and from micro- to meso-scales. The MPP has been tested for both real and artificial flow fields and it has been optimized to function with very short execution times and to give the most reasonable results under all terrain complexity and atmospheric stability conditions. DIPCOT (DIsPersion over COmplex Terrain) is a Lagrangian Puff / Particle model that has been implemented in RODOS to simulate radionuclides atmospheric dispersion over complicated terrain. For this purpose, it uses a certain number of fictitious puffs/particles which are assumed to move with the mean wind flow plus a random velocity component to simulate turbulent diffusion. The calculation of the gamma radiation dose rates in air due to the radioactive plume is calculated by a very fast method that takes into account the inhomogeneous 3-dimensional cloud shape. DIPCOT has been evaluated by comparisons to widely used real-scale experimental data sets: Copenhagen, Prairie Grass, Indianapolis and Mol. The integration of the above models greatly enhances the applicability of the RODOS system.
\end{abstract}

\section{Introduction}

Decision Support Systems (DSSs) for nuclear emergencies, such as RODOS, use atmospheric dispersion models (ADMs) to predict the spatial and temporal distribution of emitted radionuclides in the air and their deposition on the ground. One of the most important inputs required by the ADMs is meteorological information in the area of interest. Such information comes from two potential sources: measurements from the existing monitoring network and data from

1 NCSR Demokritos, Institute of Nuclear Technology and Radiation Protection, Environmental Research Laboratory, 15310 Aghia Paraskevi, Greece.

2 Department of Mechanical Engineering, University of Western Macedonia, Bakola \& Sialvera, 50100 Kozani, Greece.

3 Institute of Mathematical Machines and Systems Problems, NAS of Ukraine, Ukraine. 
Numerical Weather Prediction (NWP) models, which are usually run by meteorological services. These meteorological data need to be processed before they can be "digested" by the ADMs because (a) they do not contain all the information required by the ADMs; and/or (b) they are not gridded (in case of measurements from stations) or are gridded (in case of NWP) but on a different grid than the one used by the ADM.

The RODOS Meteorological Pre-Processor is a model that performs all the necessary actions to provide the ADMs with usable meteorological input information. It calculates missing meteorological data through empirical or semiempirical relationships, it performs spatial interpolation to calculate meteorological information on the grid that is required by the ADM, and it runs a "wind field model" to adjust the interpolated wind velocity components in order to assure mass conservation of the wind field.

There are different types of ADMs used in the RODOS DSSs. A basic requirement for a model to be applicable in nuclear emergencies is to have a short execution time. On the other hand, the level of complexity of a dispersion case that can be simulated by an ADM depends on the assumptions adopted by the model in describing atmospheric dispersion. More complex models can handle more demanding cases, such as complicated terrain or strong wind shear in the vertical direction, but require also more computer time. The approach adopted in RODOS is to incorporate ADMs of different complexity for the user to select according to the case of interest.

In order to extend the applicability of RODOS to more complex atmospheric transport and dispersion cases, the model DIPCOT (DIsPersion over COmplex Terrain) has been integrated in the system. DIPCOT is a Lagrangian puff / particle dispersion model that calculates activity concentration in air, dry and wet deposition on the ground and gamma radiation dose rate in air.

In the following sections the above mentioned models are described and their evaluation is presented.

\section{The models description}

\subsection{The RODOS Meteorological Pre-Processor}

A detailed description of the RODOS MPP is given by Andronopoulos and Bartzis (2009a). The RODOS MPP has a modular structure for ease of maintenance and up-grade: it consists of the geometrical module dealing with the computational grid and the terrain characteristics, the main module calculating all meteorological 
information by interpolation or semi-empirical relations and finally the wind flow model that adjusts the calculated horizontal velocity components and calculates a vertical velocity, taking into account the topography, to assure air flow mass conservation.

The computational grid of the RODOS MPP is 3-dimensional: it is Cartesian and uniform in the horizontal directions, with its axes aligned to the West-East and South-North directions, while it is non-uniform, terrain-following in the vertical direction. The RODOS MPP can receive input data both from meteorological stations and weather forecasting models. These are all assigned the same weight and are treated equally as "observations", either at multiple levels in the vertical (wind speed and direction, temperature, specific humidity and pressure) or at one level - surface- (precipitation intensity, net radiation, sensible heat flux, cloud cover, mixing layer height and atmospheric stability). The RODOS MPP output is a data set consisting of all the meteorological variables needed by ADMs of different types and complexities (Eulerian, Lagrangian puff / particles, simple or complex). The meteorological variables for which "observations" exist are calculated on the computational grid by spatial $1 / r^{2}$ interpolation in the horizontal direction. In the vertical direction the interpolation method depends on the variable. For the wind velocity the following interpolation schemes are used: logarithmic in the surface layer, power-law up to the mixing height, linear between observations at higher levels and geostrophic wind above the available observations. For pressure, humidity and temperature linear interpolation is used in the vertical between observations, while outside the range of observations empirical laws are used for pressure and humidity, and stability-dependent lapse rate is used for temperature. For the computation of variables non-existing in the input data sets, semi-empirical relations are used (Hanna and Chang, 1993; IAEA, 1980; Seibert et al., 1997).

The wind velocity field generated by spatial interpolation from meteorological stations or prognostic weather data does not satisfy the mass conservation principle. Therefore, a diagnostic Wind Field Model (WFM) has been implemented in the MPP which performs a divergence minimization of the wind field: it adjusts the velocity components calculated by interpolation taking into account the topography, to satisfy the mass balance in the final wind field. The WFM uses the variational approach (Sasaki, 1958, 1970; Kitada et al., 1983), which minimizes the overall differences between the initial and the final wind fields with the constraint of satisfying the continuity equation. This is achieved following the Lagrange's method that finally leads to the solution of an elliptic differential equation for the Lagrange multiplier $\lambda$. A parameter $\alpha$ that enters this equation is of particular importance, because it regulates the ratio of horizontal to vertical adjustment performed on the velocity components. In brief it is noted that 
smaller values of $\alpha$ result in more horizontal adjustment of the wind vector, while as $\alpha$ increases the horizontal adjustment is reduced. In the literature, there is no agreed or "universal" approach to calculate the value of $\alpha$ and different authors propose quite different values (Ratto et al., 1994). The most appropriate value of $\alpha$ in RODOS MPP / WFM has been determined by performing a number of wind field calculation cases over idealized and real topographies (Andronopoulos and Bartzis, 2009b). It has been established that a value of $\alpha$ close to unity, or slightly smaller gives the most reasonable results with the fastest execution times at all circumstances. For the numerical solution of the differential equation for $\lambda$, several schemes are available in the WFM. The conjugate gradient (CG) method, in combination with pre-conditioning techniques, that has been recently implemented in the WFM (Kovalets, 2006), has been proven to perform faster in all cases (Andronopoulos and Bartzis, 2009b). This method, together with other optimizations, has resulted in very short execution times for the RODOS MPP, namely in the order of a few seconds per time step.

\subsection{The atmospheric dispersion model DIPCOT}

A detailed description of RODOS-DIPCOT is given by Andronopoulos et al. (2009). In brief, atmospheric dispersion is simulated by distributing the activity of the emitted radionuclides to a certain number of fictitious puffs/particles, by calculating their displacement in time steps and by taking into account their position each time concentration is calculated. The emission rate of the fictitious puffs can be set by the user, the default value being 1 puff per 10 seconds. The puffs/particles move with the mean wind velocity at their location plus a random velocity component to simulate turbulent diffusion. The random velocity depends on the stability conditions of the atmosphere and is calculated by a Langevin equation (Thomson, 1987). The model calculates instantaneous and timeintegrated activity concentrations in air, dry and wet deposition rates and total deposition of radionuclides, gamma radiation dose rates and time-integrated dose (cloud and ground) at the locations of the RODOS dispersion grid and at locations of independent detectors.

The model uses the topography information (ground elevation and roughness) from the RODOS database, the 3-dimensional meteorological fields from the RODOS meteorological pre-processor (RODOS-MPP) and the source term (radionuclides species, emission rates, heat content, etc.) from the relevant RODOS module.

Activity concentration in air is calculated either by adding particles' activities in a certain volume or by assuming that each puff has a Gaussian spatial distribution of activity and adding the contribution of all puffs. The puffs' 
dimensions are calculated using the Taylor's (1921) theory. Turbulence statistics (velocities standard deviations, Lagrangian time scales) are parameterized on the basis of atmospheric stability, friction velocity and mixing layer height (Gryning et al., 1987; Hanna, 1982; Holtslag and Moeng, 1991). Gamma radiation dose rates in air due to radioactive cloud are calculated by adding contributions from all puffs, in order to take into account the real inhomogeneous plume shape. To save computational time the above 3-dimensional integral has been transformed to 1dimensional integral and its values have been pre-calculated and stored for a wide range of parameters (puffs distances and dimensions) covering most probable values. This file is read at the beginning of the run and then, during the run, linear interpolation is performed to calculate the exact value when it is needed. For calculating gamma radiation dose rates due to deposited material on the ground, the infinite plane assumption is adopted, based on the dimensions of the RODOS dispersion grid cells.

\section{Model validation}

The modelling system RODOS-MPP-DIPCOT has been validated in a first stage by simulating real-scale dispersion experiments that are widely used by the scientific community for model-validation purposes: Copenhagen, Prairie Grass, Indianapolis and Mol experiments. The validations have been performed by comparing model-calculated to experimental values of dispersion-related parameters: maximum concentrations, crosswind integrated concentrations and plume crosswind dimensions. Statistical indices (e.g., fractional bias, normalized mean square error, factor-of-2, etc.) have been calculated for the Inidianapolis experiments. In the Mol - Argon-41 experiments, fluence rates in air have been compared, validating thus the gamma radiation dose rate method. Details can be found in Andronopoulos et al. (2009). Here some indicative comparisons between model-calculated and experimental data are presented in Figures 1-3, concerning plume width, maximum concentrations and fluence rate in air. Based on these, but also on the rest of the results, the modelling-system performance is satisfactory. It must be noted that the above cases concern dispersion over flat terrain. Simulations of dispersion cases in complicated terrain will follow at a later stage, since such data are rather scarce.

\section{Conclusions}

The RODOS Meteorological Pre-Processor (MPP) is a model that receives as input meteorological data from monitoring stations and weather prediction models and produces as output a meteorological data set suitable for driving a variety of Atmospheric Dispersion Models. The MPP includes a Wind-Field Model in order 


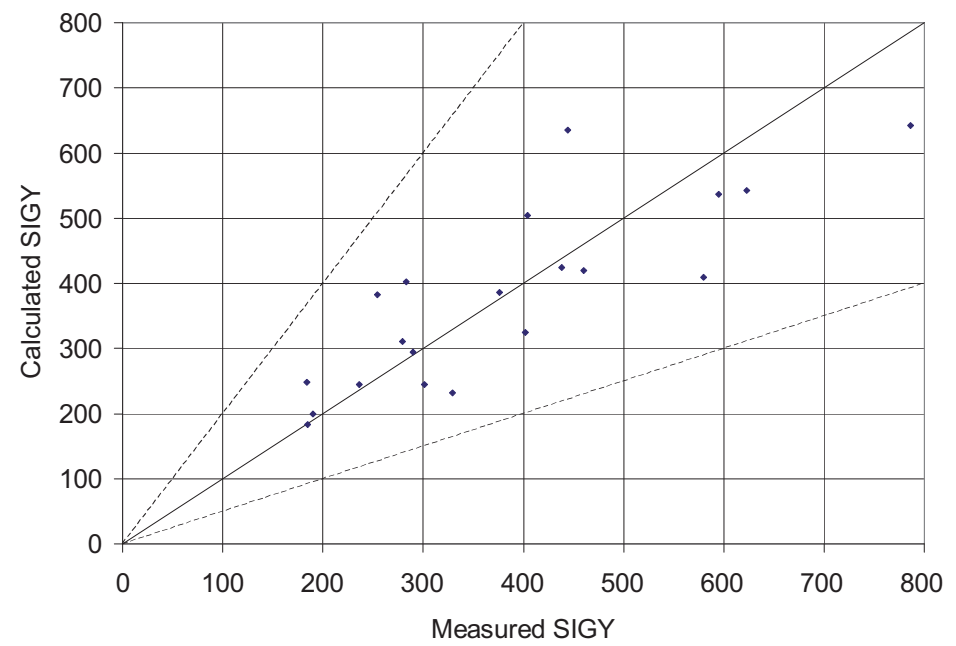

Figure 1 - RODOS-DIPCOT validation for Copenhagen experiments: scatter plot of calculated vs. measured plume width.

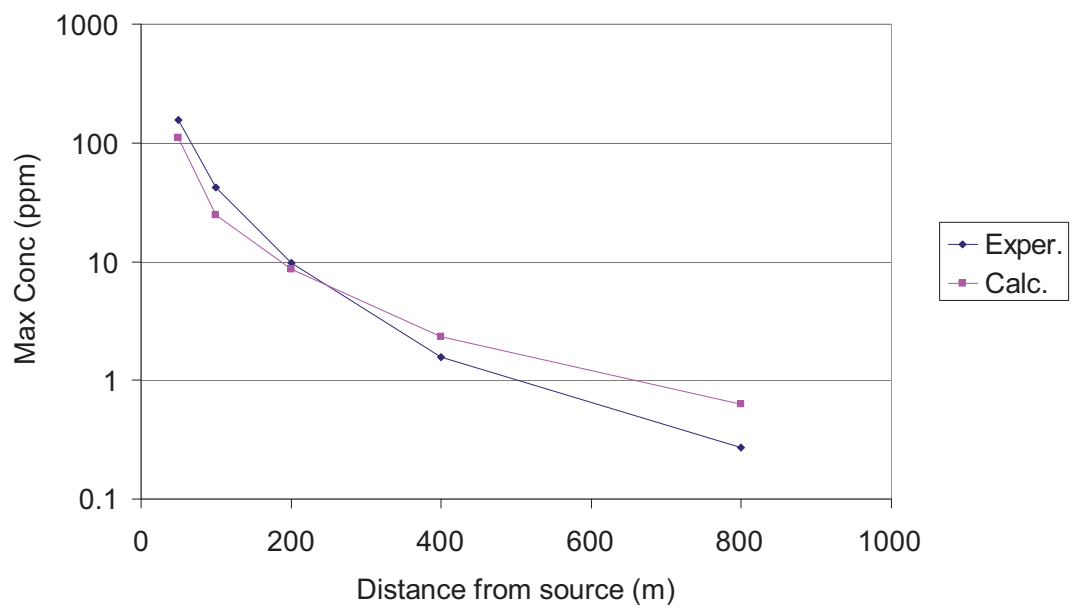

Figure 2 - RODOS-DIPCOT validation for Prairie Grass experiment: variation of calculated and measured maximum concentrations (right) with distance from source.

to calculate a mass consistent 3-dimensional wind field, taking into account terrain complexities. The atmospheric dispersion model DIPCOT has been implemented in RODOS to be applied in cases of complicated terrain or meteorological 


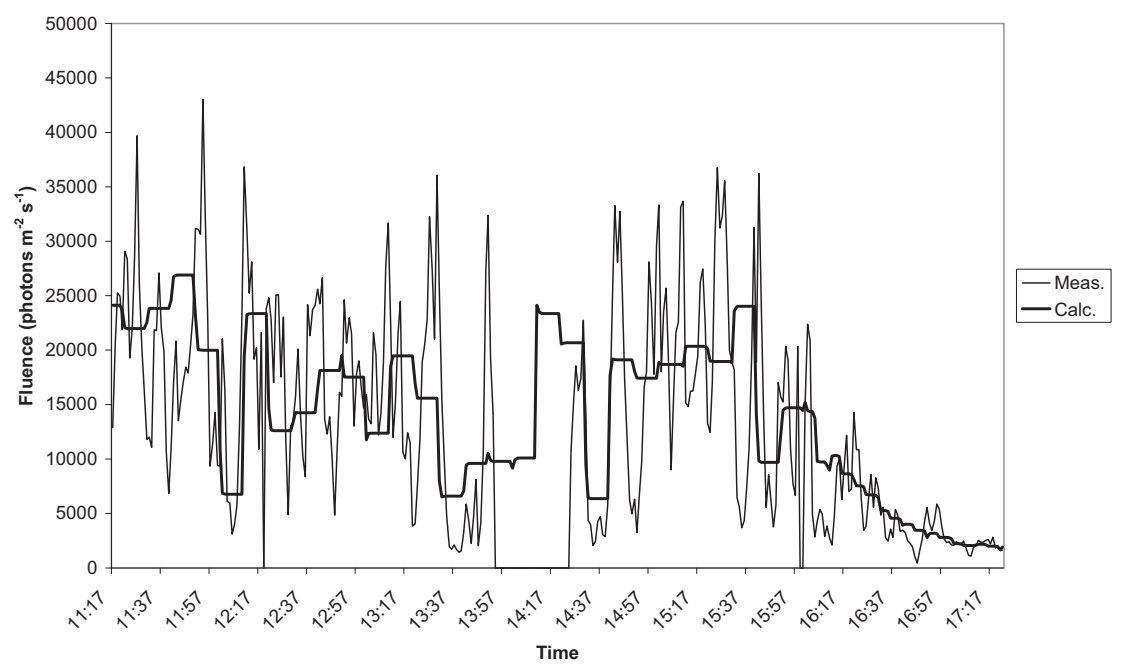

Figure 3 - RODOS-DIPCOT validation for Mol-Argon-41 experiments: time-history of calculated and measured fluence rates.

conditions. It is a Lagrangian puff / particle model with a random motion of the particles in addition to the mean wind velocity. DIPCOT incorporates a novel and fast model for calculating gamma radiation dose rates from a 3-dimensional inhomogeneous cloud. The modelling system consisting of the RODOS MPP and DIPCOT has been validated by comparing calculated to measured dispersionrelated parameters for well known experimental cases (Copenhagen, Prairie Grass, Indianapolis and Mol experiments). Qualitative (plots) and quantitative (statistical) means have been used for validation and the results are in general satisfactory. Further validation exercises are underway for cases of dispersion over complex terrain. The integration of the above models in the RODOS system has substantially enhanced its range of applicability.

Acknowledgement. This work has received partial financial support from the European Commission Sixth Framework Programme (Nuclear Fission/Radiation Protection) under the EURANOS integrated project: European approach to nuclear and radiological emergency management and rehabilitation strategies (Contract No: FI6R-CT-2004-508843).

\section{REFERENCES}

Andronopoulos S., Bartzis J.G. (2009a) Model description of the RODOS Meteorological PreProcessor, Report RODOS(RA2)-TN(09)02, available in http://www.rodos.fzk.de/rodos.html. 


\section{S. ANDRONOPOULOS et al.}

Andronopoulos S., Bartzis J.G. (2009b) Report on performance tests of the mass-consistent wind-field model, Report EURANOS(CAT2)-TN(09)04.

Andronopoulos S., Davakis E., Bartzis J.G. (2009) RODOS-DIPCOT Model Description and Evaluation, Report RODOS(RA2)-TN(09)01, available in http://www.rodos.fzk.de/rodos.html.

Gryning S.E., Holstag A.A.M., Irwin J.S., Sivertsen B. (1987) Applied dispersion modelling based on meteorological scaling parameters, Atmos. Environ. 21, 79-89.

Hanna S.R. (1982) Atmospheric turbulence and air pollution modelling. In: Atmospheric Turbulence and Air Pollution Modelling (Nieuwstadt F.M.T., van Dop H., Ed.) pp. 275-310, D. Reidel, Dordrecht, Holland.

Hanna S.R., Chang J.C. (1993) Hybrid plume dispersion model (HPDM) improvements and testing at three field sites, Atmos. Environ. 27A, 1591-1508.

Holtslag A.A.M., Moeng C. H. (1991) Eddy diffusivity and countergradient transport in the convective atmospheric boundary layer, J. Atmos. Sci. 48, 1690-1998.

IAEA (1980) Atmospheric Dispersion in Nuclear Power Plant Siting. International Atomic Energy Agency, Safety Series, 50-SG-S3.

Kitada T., Kaki A., Ueda H., Peters L.K. (1983) Estimation of vertical air motion from limited horizontal wind data-a numerical experiment, Atmos. Environ. 17, 2181-2192.

Kovalets I. (2006) RODOS System Meteorological and Atmospheric Dispersion Module functionality enhancement by introduction of numerically efficient algorithms. Final Report of the "RODOS / METADM - enhance" Project Contract No 516492 (FI6R).

Ratto C.F., Festa R., Romeo C., Frumento O.A., Galluzzi M. (1994) Mass-consistent models for wind fields over complex terrain: The state of the art, Environ. Softw. 9, 247-268.

Sasaki Y. (1958) An objective analysis based on the variational method, J. Met. Soc. Japan 36, 77-88.

Sasaki Y. (1970) Some basic formalism in numerical variational analysis, Mont. Weather Rev. 98, 875-883.

Seibert P., Beyrich F., Gryning S.-E., Joffre S., Rasmussen A., Tercier Ph. (1997) Mixing Height Determination for Dispersion Modelling, Report of Working Group 2. In: COST Action $710_{-}$ Final Report, EUR 18195 EN. European Commission.

Taylor G.I. (1921) Diffusion by continuous movements, Proc. Lond. Math. Soc. 20, 169-211.

Thomson D.J. (1987) Criteria for the selection of stochastic models of particle trajectories in turbulent flows, J. Fluid Mech. 180, 529-556. 